\title{
PSYCHOSOCIAL EFFECTS OF PCOS ON REPRODUCTIVE-AGE WOMEN; A PRELIMINARY EXPLORATORY STUDY BASED IN KERALA
}

\author{
Reshma Ramesh ${ }^{1}$ and Swati Dinesh ${ }^{2}$ \\ ${ }^{1}$ Center for Women Empowerment and Gender Equality, Amrita Vishwa Vidyapeetham, Kollam, 690525 India \\ ${ }^{2}$ Master's in Social Work Candidate, Amrita Vishwa Vidyapeetham, Kollam, 690525 India
}

Corresponding author: Reshma Ramesh ${ }^{1}$

Email: reshma.ramesh@ammachilabs.org

\begin{abstract}
Polycystic ovary syndrome (PCOS) is a hormonal disorder found in reproductive age women without a finite cause of existence. While clinical aspects of the study attribute itself to a more significant part of the research, there is also a need to understand patients' perception of better design interventions. The purpose of this study was to perform a preliminary qualitative study to analyse and apprehend the feelings, expressions, perceptions, and experiences expressed by patients associated with the PCOS. The study exercised in-depth interviews with women between 18 to 35 years in Kollam district, Kerala, India. The study results reveal that psychosocial aspects such as lifestyle changes, coping mechanisms, attitudes and beliefs, awareness levels, and physician-patient relations are affecting women among this population. The study concluded that for any behavioural intervention to work for women affected by PCOS, it is essential to take into account the aforementioned psychosocial aspects.
\end{abstract}

Keywords: Polycystic Ovary Syndrome;Psychosocial effects;PCOS;Patient Quality of life

\section{INTRODUCTION}

PCOS or Polycystic Ovary Syndrome is known to be a hormonal disorder were ovaries enlarge, and small cysts are found on its outer surface. Literature indicates that about $8 \%$ to $20 \%$ of women in their reproductive age are affected by PCOS ${ }^{1}$. Women diagnosed with PCOS were predominantly in their $20 \mathrm{~s}$ or $30 \mathrm{~s}$, but studies have shown that PCOS may affect girls even before they reach menstruation ${ }^{2}$. The Rotterdam consensus workshop in 2003, concluded that Polycystic syndrome is a syndrome of ovarian dysfunction ${ }^{3}$. The clinical manifestation of the condition includes menstrual irregularities, signs of excess androgens, weight management issues, insulin resistance, and elevated serum LH levels ${ }^{3}$. Well recognized literature in the PCOS arena suggests that lifestyle changes like a highcalorie diet, lack of exercise, and insulin resistance are leading causes ${ }^{4}$. Research also revealed that that genetic predisposition might affect the exposure of women to PCOS ${ }^{5}$. While diagnostic criteria are limited to three groups (NIH/NICHD, Rotterdam criteria, and Androgen Excess), the syndrome is currently known to be a genetically complex endocrine disorder of uncertain aetiology with complicated pathophysiology ${ }^{6}$. Women with PCOS have been seen with hormonal imbalance, and problems relate to metabolism. It has an overall effect on the health and appearance of women.

Prevalence rates studies on PCOS form most of the literature, and they sate it as highly variable. Global prevalence rates explained in the literature vary from $8 \%$ to $20 \%(6,7,8,9,27)$. While globally, the syndrome has gained momentum in research, prevalence rates studies in Asian Scenario is less ${ }^{10}$. Predominant studies in the literature concerning PCOS in India are based on quantitative techniques to determine prevalence rates and disease epidemiology. Studies on oxidative stress, vitamin $D$ deficiencies, obesity among adolescents have been studies on an Indian perspective ${ }^{(11,12,13)}$. Globally, the psychoanalytic approach was undertaken in older literature ${ }^{14}$. Jones et al. (2008), found through his systematic review of literature, that only nineteen papers till the date of the study were conducted connecting QoL of patients with polycystic ovaries syndrome ${ }^{15}$.

One of the primary studies in Psychological arena was by Trent et al. (2002) using the Child Health Questionnaire (CHQ- CF87) showed that HealthRelated Quality of Life scores of adolescents with PCOS was significantly lower from patients without PCOS in four of the twelve areas assessed by the questionnaire: general health perceptions, physical functioning, behaviour, and family activities ${ }^{16.26}$. The Effect of Body Mass Index on QoL disturbances in adolescents was studied by Trent et al. (2005) ${ }^{17,18}$. The only Indian Study on Psychosocial aspects of PCOS is by PG Sundararaman., et al. (2008), where the author mentioned that women with PCOS had increased phycological distress ${ }^{19}$.

The current paper discusses a preliminary exploratory study among postgraduate girl students and working women diagnosed with PCOS within the age group of 18 to 35 and located in the Kollam District of Kerala, India. 
The study focused on understanding the different psychosocial effects i.e.; relations PCOS has to the interrelations of social factors and individual thoughts and behaviour. The primary motivation behind the study was to provide concise literature on the PCOS condition from a social perspective and in a local context. The paper approached a qualitative technique wherein semi-structured in-depth interviews were conducted with respondents, following the process of obtaining their informed consent for their participation. Participants were asked to share their experiences related to the psychosocial aspects of PCOS and the effect the diagnosis has had on their health and well-being. The study's self-reported themes not only captured the rational expressions and attitudes women have towards PCOS but also their expectations and concerns towards future life.

\section{METHODOLOGY}

The population under the study consisted of women between 18 to 35 years with PCOS, who is either a student or working group in Kollam District, Kerala India. The population was a purposive sampling recruited from a support group of working women and college students with PCOS. Participants were from an educational background ranging from graduation to doctoral students. Twelve respondents were from the age group from 25 to 35,13 were from the age group 18 to 24 . All these respondents were from an urban setting and had access to health care. The diagnosis period ranged in participants from 1.5 months to 10 years. 30\% of the respondents were married, and the rest were single. The population varied in caste, cultural and socio-economic status.

Information on the type of study was provided to respondents before seeking their consent. Interviews were conducted only with the respondents, who had volunteered and given consent to take part in the study. In-depth interviews were conducted over one and a half months, and each interview session was approximately one and a half hours long. Interviews were arranged at a place and time convenient for the participant. The interview questions were designed for developing an understanding of how women who have been diagnosed with PCOS, perceived themselves as living with PCOS, their emotional response to the condition feel, symptoms they self-reported, lifestyle habits and the psychosocial effects the condition has had on their life and relationships with peers and family. The respondents were also asked about the different treatment options they practice and their satisfaction related to the treatment provided. Interviews were conducted in the participants' and authors' native language of Malayalam. The interviews were transcribed, and the transcription was translated into English.
A conventional content analysis technique was used to derive themes related to attitudes and psychosocial effects on PCOS among respondents. Interview transcripts were initially analysed and identified for small meaningful segments from the transcripts, which were relevant to the topic. The recognized meaningful units from the transcripts were then transferred into rows in an excel sheet. Units with similar ideas and descriptions were then highlighted to form codes. The codes with similar meaning are color-coded and then grouped to capture subcategories. With the emerging subcategories, themes related to different psychosocial effects of the syndrome and its effects on the respondent's lifestyle and quality of life were recorded as results.

\section{RESULTS}

Major themes that emerged from Content Analysis were;

- Lifestyle changes and choices

- Attitude towards daily routine and diagnosis

- Bodyweight issues

- Fertility concerns and psychological constructs

- Role of Family and Friends in social stress

- Beliefs and misconceptions related to PCOS

- Patient-physician relationships.

\section{Lifestyle changes and choices}

Women shared that they consider lifestyle changes as the very first step towards conquering PCOS. Maintaining a healthy lifestyle was a choice that the participant's families wanted them to follow; however, the women mentioned that they are often more unhealthy when they are with their peers. Awareness levels of the syndrome's condition and diagnosis were much higher among working women than among students. A particular focus on food habits and routine exercise were mentioned by respondents when asked about post PCOS diagnosis measures a woman should take to reduce symptoms. Respondents shared the following during interviews:

"I started concentrating more on healthy foods than unhealthy ones."

"Understood the need for exercises in daily routine as I felt tired easily due to inactivity on a day to day basis."

\section{Attitude towards daily routine and treatment}

Working women sample in the study analysis, mentioned that they find it difficult to cope with a routine diet and exercise due to work schedules. Students reported that it was difficult 
for them to follow a strict regimen, along with their class routines and hostel life. Respondents often mentioned that it was necessary to have a group of people perform these tasks along with them as they felt demotivated to perform actions related to diet and exercise when alone. Irregularities in periods were considered normal until they were aware of the ill effects of the condition. When it comes to diagnosis, mixed responses were received. The population included women and girls who have undergone diagnosis were under medication. $5 \%$ of the population believed that the condition would get better without undergoing medication. The study probed them further to understand if the nomedication step is attributed to a lack of interest or awareness about the syndrome. The respondent's preference for future life played an important role in taking up treatments. People who had marriage were open to treatment, while those respondents who did not plan for it were reluctant.

\section{Bodyweight issues}

About $95 \%$ of the respondents mentioned having weight-related issues concerning PCOS. Many found it challenging to maintain the weight that they desire. Women responded that they were asked to regulate their weight post-diagnosis as being overweight can exacerbate the PCOS symptoms. The participants mentioned that society rejects women who are overweight and struggling with their weight causes them much anxiety.

"There is an increase in pressure from society and relatives to reduce your weight; otherwise, it is difficult to get good relationships. Women with bodyweight are considered unattractive."

One of the women's opinion on weight management issue below

"I know how hard it is to continue on a strict exercise when you see others have an easy life. However, since I know it can lead to further complications, it gives the motivation to change my mental inhibitions related to workouts."

This suggests that personal decision have more power, is seen decisions when connected to intrinsic motivation, along with support from external sources.

\section{Fertility concerns and psychological constructs}

Fertility fears and constraints within social life were mentioned by the working women population, while none of the students mentioned that they were bothered about fertility issues. One of the participants expressed her fear;
"Even in my social life, I have faced questions about pregnancy. Sometimes I fear that I may also face fertility issues without solving my PCOS issues."

The student population did seem concerned about fertility, perhaps because they lack awareness of the potential effects of PCOS on future family planning.

\section{Role of Family and Friends in dealing with Social Stress}

This study revealed that stress-related to PCOS conditions in women could be reduced with family and peer support. PCOS support groups and helpful peers helped the participants in this study to gain the confidence that they are not alone and a secured base for them to contact in need. As extremely helpful, family support in all endeavours related to treatment and pre and post-diagnosis were mentioned by respondents. While Immediate family responses were positive towards women diagnosed with this syndrome, extended families put pressure on the women over their condition. One of the respondents stated the following.

"I used to eat meat as a part of the daily diet. Post my diagnosis, and I stopped eating meat in order to avoid high-fat foods. It is difficult for my mom to cook special dishes for me, but she willingly does this like every mother. However, during family occasions, it is difficult to tell people the reason for not having meat."

Married women face an increase in stress levels related to family planning because culturally, women are expected to have children as soon as possible after marriage. One of the respondents mentioned

"I have seen other married women getting stressed or pressured about having babies. However, there is no awareness in the people who pressure a person having PCOs/ People think having babies makes life complete."

Women also responded that decision making related to whether or not they should have children mainly rests with their family members, and couples are likely to follow their family's wish.

\section{Beliefs and Awareness levels related to PCOS}

The participants mentioned that the advancement of technologies and varied treatment options lessen the stigma associated with the PCOS. Friends and family have started discussing it openly when it comes to diagnosis and treatment. The following beliefs were reported by women related to PCOS, 
"It is a myth that ladies with PCOS cannot conceive, during the time you do not plan to conceive, birth control pills are a good option to regulate PCOS, regular periods with minimal side effects."

"There are many misconceptions regarding PCOS I feel like, women will not conceive, the cyst is cancerous. Each case is different from others as far as I know. So it's important to stick on to the regime doctor suggests. After all, they know the best."

"Physicians suggest that PCOS will be cured after conceiving, but in my case, PCOS was still prevalent after my delivery. It was tough for me to cope with the menstrual irregularities."

One of the respondents mentioned that in the initial years after she began menstruating, physicians believed and confirmed that irregularity would be believed to resolve with age, so if proper diet and exercise were prescribed earlier, she wouldn't have to face more severe consequence in future.

\section{Physician-Respondent relationships}

One of the themes that participants mentioned, especially the students in the study population, was the importance of a good and open relationship with the physician. Participants mentioned that they prefer female doctors when it comes to sharing problems related to PCOS. Also, a relationship based on trust should be built in order to have the motivation to make severe lifestyle changes. Women mentioned they are more likely to stick on to a daily routine when they get support from their physicians, especially the mental stress they face due to their PCOS condition. One of the respondents mentioned the following

"Sometimes discussing needs related to PCOS, different feelings, with family and friends creates tension and it would be good to have a doctor to speak about problems openly"

"It is difficult to go for consultation as my doctor always scolds me for not maintaining my weight and not following a regular diet. I wish I had more support from the doctor"

\section{DISCUSSION}

The study was aimed to explore the different psychological and social impact, women with PCOS condition phases in their day to day life. Clinical manifestation of the condition is well researched in literature and has its own characteristics causes; the current article explains self-reported emotional stress, awareness levels and social experiences women with PCOS condition faces.

The study analysis supports the existing literature related to lifestyle changes ${ }^{4}$. The choice of a healthy and active lifestyle emerged as a common theme among the participants. Having balanced protein and carbohydrate intake and reducing fast foods where changes mentioned related to food intake, while regular exercise, practicing yoga, maintaining blood glucose level were other responses related to lifestyle changes. The respondents mentioned that these choices were brought about intentionally because they were asked to follow the healthier practices by a physician or family member. Following a strict routine related to diet and exercise are necessary for resolving the PCO Syndrome 20,21 . Respondents in the study, however, mentioned that this is one of the toughest jobs to carry forward in the social and physical environment that they live.

Consistent with the existing research, body weight issues were also reported in the study. The literature also mentioned that weight issue upsets self-confidence of a person, social and emotional well-being, therefore impacting HRQoL in PCOS adolescents ${ }^{22}$. Increasing body weight may lead to diabetes in women as they age $^{23}$. The respondents in the study also stated about bodyweight issues. 50\% of the sample reported taking up a positive measure to control their body weights; the other half of the population did not consider it essential or demonstrate any efforts in this. Most women wished to accomplish a weight they coveted but was unable to cope up with the growing requirement for self-disciple to carry forward a strict regime. The lack of achievement related to often leads to introversion and social withdrawal.

Studies have mentioned that infertility caused by PCOS leads to a significant amount of stress ${ }^{17}$. The participants mentioned that women who have infertility issues are often stigmatized in their society. In their society, even with high health awareness, expectations from a woman, particularly marriage practices and family structure, are not equal. While awareness levels are high among the population, implementing them in their daily life's was a constraint reported by the majority of the respondents, The leading cause of this was social pressure in leading a different lifestyle than their peers. Social support is needed when it comes to coping with physical and psychological stress $^{24}$.

The literature suggests that it is essential to have a multi-disciplinary approach while treating $\mathrm{PCOS}^{25}$. Bringing in the factor of care, referring to a proper dietitian and endocrinologist, and even mental health specialists can help patients with PCOS have increased bonds with physicians and more likely to open up about their intimate problems related to the syndrome and therefore get better treatment. A PCOS support group also plays a vital role in treating patients' isolation 
and increasing awareness of how best to treat PCOS. The majority of the study results are harmonious with existing literature, which states that PCOS strongly impacts the psychosocial aspects of a patient's life. For an effective intervention design, the factors explained in the study will provide us with areas where proper awareness, access, and information can be provided.

\section{CONCLUSION}

The study examined the influence of Polycystic ovarian syndrome has on a women's psychological development. The results are formulated based on the self-reports participants shared during in-depth interviews. The findings from the study state that several factors within women and around her affect the acceptance, diagnosis, and treatment of PCOS condition. Their Lifestyle choices and practice play a vital role in managing the syndrome. Family and Friends play an essential role in supporting and promoting healthy behaviour in women. Above all, women's attitude towards the syndrome and the right knowledge about the condition and beliefs form most of the psychosocial aspects of this disease. The study concluded supporting that for any behavioural intervention to work for women affected by PCOS; it is essential to also take into account the psychosocial aspects to increase the patient's participation. While this study is limited in these context-specific results, future studies are required to see how integrating this body of knowledge can help in health-promoting interventions related to PCOS.

\section{ACKNOWLEDGEMENTS}

This research wouldn't be possible without the guidance and support received from Sri Mata Amritanandamayi Devi, Chancellor of Amrita Vishwa Vidyapeetham. We would like to thank field staff, academicians and faculties at Amrita Vishwa Vidyapeetham for their support and sincere effort in providing the right kind of support at the right time.

\section{REFERENCES}

1. Sirmans SM, Pate KA. Epidemiology, diagnosis, and management of polycystic ovary syndrome. Clinical epidemiology. 2014;6:1.

2. Matzke A. The Underdiagnosis of Polycystic Ovarian Syndrome in Normal Weight Adolescent Females.

3. Rotterdam ESHRE/ASRM-Sponsored PCOS Consensus Workshop Group. Revised 2003 consensus on diagnostic criteria and long-term health risks related to polycystic ovary syndrome (PCOS).
Human reproduction. 2004 Jan $1 ; 19(1): 41-7$.

4. Holte J, Gennarelli G, Wide L, Lithell H, Berne C. High prevalence of polycystic ovaries and associated clinical, endocrine, and metabolic features in women with previous gestational diabetes mellitus. The Journal of Clinical Endocrinology \& Metabolism. $1998 \mathrm{Apr}$ $1 ; 83(4): 1143-50$.

5. Jahanfar S, Eden JA. Genetic and nongenetic theories on the etiology of polycystic ovary syndrome. Gynecological Endocrinology. 1996 Jan 1;10(5):357-64.

6. Bharathi RV, Swetha S, Neerajaa J, Madhavica JV, Janani DM, Rekha SN, Ramya S, Usha B. An epidemiological survey: Effect of predisposing factors for PCOS in Indian urban and rural population. Middle East Fertility Society Journal. 2017 Dec 1;22(4):313-6.

7. Azziz R, Woods KS, Reyna R, Key TJ, Knochenhauer ES, Yildiz BO. The prevalence and features of the polycystic ovary syndrome in an unselected population. The Journal of Clinical Endocrinology \& Metabolism. 2004 Jun $1 ; 89(6): 2745-9$.

8. Knochenhauer ES, Key TJ, Kahsar-Miller $M$, Waggoner W, Boots LR, Azziz R. Prevalence of the polycystic ovary syndrome in unselected black and white women of the southeastern United States: a prospective study. The Journal of Clinical Endocrinology \& Metabolism. 1998 Sep 1;83(9):3078-82.

9. Asunción M, Calvo RM, San Millán JL, Sancho J, Avila S, Escobar-Morreale HF. A prospective study of the prevalence of the polycystic ovary syndrome in unselected Caucasian women from Spain. The Journal of Clinical Endocrinology \& Metabolism. 2000 Jul 1;85(7):2434-8.

10. Nidhi R, Padmalatha V, Nagarathna R, Amritanshu R. Prevalence of polycystic ovarian syndrome in Indian adolescents. Journal of pediatric and adolescent gynecology. 2011 Aug 1;24(4):223-7.

11. Raj M, Kumar RK. Obesity in children \& adolescents. The Indian journal of medical research. 2010 Nov;132(5):598.

12. Indhumol TD, Pavithran S, George LK. Effectiveness of Structured Teaching Program On Knowledge Regarding Prevention Of Urinary Tract Infection Among Adolescent Girls. International 
Journal of Pharma Medicine and Biological Sciences. 2014 Jul 1;3(3):121.

13. Sumithra NU, Lakshmi RL, Menon NL, Subhakumari KN, Sheejamol VS. Evaluation of oxidative stress and hsCRP in polycystic ovarian syndrome in a tertiary care hospital. Indian journal of clinical biochemistry. $2015 \mathrm{Apr}$ $1 ; 30(2): 161-6$.

14. Nesbitt Jr RE, Rollender M, Fisher S, Osofsky HJ. Psychologic correlates of the polycystic ovary syndrome and organic infertility. Fertility and Sterility. 1968 Sep 1;19(5):778-86.

15. Jones GL, Hall JM, Balen AH, Ledger WL. Health-related quality of life measurement in women with polycystic ovary syndrome: a systematic review. Human reproduction update. 2008 Jan $1 ; 14(1): 15-25$.

16. Trent ME, Rich M, Austin SB, Gordon CM. Quality of life in adolescent girls with polycystic ovary syndrome. Archives of pediatrics \& adolescent medicine. 2002 Jun 1;156(6):556-60.

17. Trent ME, Rich M, Austin SB, Gordon CM. Fertility concerns and sexual behavior in adolescent girls with polycystic ovary syndrome: implications for quality of life. Journal of pediatric and adolescent gynecology. 2003 Feb 1;16(1):33-7.

18. Trent M, Austin SB, Rich M, Gordon CM. Overweight status of adolescent girls with polycystic ovary syndrome: body mass index as mediator of quality of life. Ambulatory Pediatrics. 2005 Mar 1;5(2):107-11.

19. Sundararaman PG, Sridhar GR. Psychosocial aspects of women with polycystic ovary syndrome from south India. The Journal of the Association of Physicians of India. 2008 Dec 1;56:945-8.

20. Norman RJ, Mahabeer S, Masters S. Ethnic differences in insulin and glucose response to glucose between white and Indian women with polycystic ovary syndrome. Fertility and sterility. 1995 Jan 1;63(1):58-62.

21. Norman RJ, Davies MJ, Lord J, Moran LJ. The role of lifestyle modification in polycystic ovary syndrome. Trends in Endocrinology \& Metabolism. 2002 Aug 1;13(6):251-7.

22. Crouch M, McKenzie H. The logic of small samples in interview-based qualitative research. Social science information. 2006 Dec;45(4):483-99.

23. Lauenborg J, Mathiesen E, Hansen T, Glumer C, Jørgensen T, Borch-Johnsen K, Hornnes $P$, Pedersen $O$, Damm P. The prevalence of the metabolic syndrome in a Danish population of women with previous gestational diabetes mellitus is three-fold higher than in the general population. The Journal of Clinical Endocrinology \& Metabolism. 2005 Jul 1;90(7):4004-10.

24. Coyne JC, Downey G. Social factors and psychopathology: Stress, social support, and coping processes. Annual review of psychology. 1991 Feb;42(1):401-25.

25. Kaczmarek C, Haller DM, Yaron M. Health-related quality of life in adolescents and young adults with polycystic ovary syndrome: a systematic review. Journal of pediatric and adolescent gynecology. 2016 Dec $1 ; 29(6): 551-7$

26. Lewis PR, Travers CA, Esler DM, Guttikonda K, Dixon A. The psychosocial experience of women with PCOS: a case control study. Australian family physician. 2007 Nov;36(11):965.

27. Moran L, Gibson-Helm M, Teede H, Deeks A. Polycystic ovary syndrome: a biopsychosocial understanding in young women to improve knowledge and treatment options. Journal of Psychosomatic Obstetrics \& Gynecology. 2010 Mar 1;31(1):24-31. 